\title{
A novel surgical marking system for small peripheral lung nodules based on radio frequency identification technology: Feasibility study in a canine model
}

Fumitsugu Kojima, MD, ${ }^{\mathrm{a}, \mathrm{b}}$ Toshihiko Sato, MD, PhD, ${ }^{\mathrm{b}}$ Hiromi Takahata, MEng, ${ }^{\mathrm{c}}$ Minoru Okada, $\mathrm{PhD},{ }^{\mathrm{d}}$ Tadao Sugiura, $\mathrm{PhD},{ }^{\mathrm{d}}$ Osamu Oshiro, $\mathrm{PhD},{ }^{\mathrm{c}}$ Hiroshi Date, $\mathrm{MD}, \mathrm{PhD},{ }^{\mathrm{b}}$ and Tatsuo Nakamura, MD, $\mathrm{PhD}^{\mathrm{a}}$

Objective: We investigated the feasibility and accuracy of a novel surgical marking system based on radiofrequency identification (RFID) technology for the localization of small peripheral lung nodules (SPLNs) in a canine model.

Methods: The system consists of 4 components: (1) micro RFID tags (13.56 MHz, $1.0 \times 1.0 \times 0.8 \mathrm{~mm}),(2)$ a tag delivery system with a bronchoscope, (3) a wand-shaped locating probe (10-mm diameter), and (4) a signal processing unit with audio interface. Before the operation, pseudolesions mimicking SPLNs were prepared in 7 dogs by injecting colored collagen. By use of a computed tomographic (CT) guide, an RFID tag was placed via a bronchoscope close to each target lesion. This was then followed by scanning with the locating probe, and wedge resection was performed when possible. Operators can locate the tag by following the sound emitted by the system, which exhibits tone changes according to the tag-probe distance. The primary outcome measure was the rate of wedge resection with good margins.

Results: A total of 10 pseudolesions imitating SPLNs were selected as targets. During thoracoscopic procedures, 9 of 10 tags were detected by the system within a median of 27 seconds. Wedge resections were performed for these 9 lesions with a median margin of $11 \mathrm{~mm}$. The single failure was caused by tag dislocation to the central airway.

Conclusions: Successful localization and wedge resection of pseudolesions with appropriate margins were accomplished in an experimental setting. Our RFID marking system has future applications for accurately locating SPLNs in a clinical setting. (J Thorac Cardiovasc Surg 2014;147:1384-9)

Video clip is available online.

The accurate localization of small peripheral lung nodules (SPLNs) in a thoracoscopic setting is a challenging task, although video-assisted thoracic surgery is a more suitable approach than open surgery for small lesions. ${ }^{1}$ In accordance with evolving patient and social demands, the

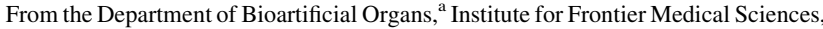

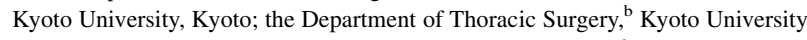
Hospital, Kyoto; the Graduate School of Engineering Science, ${ }^{\mathrm{c}}$ Osaka University, Toyonaka; and the Graduate School of Information Science, ${ }^{\mathrm{d}}$ Nara Institute of Science and Technology, Ikoma, Japan.

This project was supported by grants from The Japanese Foundation for Research and Promotion of Endoscopy, and Japan Society for the Promotion of Science Fujita Memorial Fund for Medical Research.

Disclosures: Authors have nothing to disclose with regard to commercial support.

Received for publication March 7, 2013; revisions received May 15, 2013; accepted for publication May 31, 2013; available ahead of print July 15, 2013.

Address for reprints: Toshihiko Sato, MD, PhD, Department of General Thoracic Surgery, Kyoto University, 54 Shogoin, Kyoto 6068507, Japan (E-mail: tsato@kuhp kyoto-u.ac.jp)

0022-5223/\$36.00

Copyright (c) 2014 by The American Association for Thoracic Surgery http://dx.doi.org/10.1016/j.jtcvs.2013.05.048 importance of minimally invasive surgical procedures has increased in recent years. In 2010, the rate of videoassisted thoracic surgery reportedly reached nearly $60 \%$ of lung cancer surgery in $\mathrm{Japan}^{2}$ and $44.7 \%$ in the United States. ${ }^{3}$ The vast majority of SPLNs are therefore likely to be resected by a thoracoscopic approach in Japan.

Many techniques to assist the localization of SPLNs have been developed and reported; these include finger palpation, percutaneous hook-wire placement, preoperative dye marking, fluoroscopy with a contrast medium, intraoperative ultrasonography (US), and radiotracer-guided surgery. ${ }^{4}$ These techniques have proved reliable to a certain extent, but all possess considerable problems in complications, cost, technical difficulty, and availability of facilities. ${ }^{5}$ Consequently, there is not yet an established "best technique," and the choice of method is highly dependent on each surgeon's preference.

Despite the lack of a definitive solution for determining SPLN location, thoracic surgeons are required to manage an increasing number of small pulmonary nodules owing to the continued improvement of computed tomography (CT) technology. In 2011, the National Lung Screening Trial established the ability of low-dose CT screening to reduce mortality in a high-risk population. ${ }^{6}$ Also, recent guidelines for lung cancer screening from The American 


\section{Abbreviations and Acronyms \\ $\mathrm{CT}=$ computed tomography \\ RFID $=$ radiofrequency identification \\ SPLN $=$ small peripheral lung nodule \\ US = ultrasonography}

Association for Thoracic Surgery have recommended the surgical excision of subcentimeter lesions in patients whose tumors show suspicious changes in size or appearance. ${ }^{7}$ For years, the preoperative marking for lung nodules smaller than $10 \mathrm{~mm}$ in size has also been recommended. ${ }^{8}$ In light of these advances in CT screening and recommendations, there is an unprecedented need to improve localization techniques.

Furthermore, in this era of minimally invasive surgery, there is an increasing demand for innovative localization techniques. An ideal method not only would ensure patient safety but also would allow for adequate minimum margins from the lesion. For instance, maintenance of appropriate margins for sublobar curative resection of primary lung cancer has been shown to reduce recurrences. ${ }^{9}$ The importance of appropriate margins has similarly been emphasized in the resection of metastatic pulmonary nodules to prevent local recurrence. ${ }^{10}$ However, for surgeons to ensure adequate margins, they must first be able to gauge the exact distance from the target lesion to the cutting line for resection.

To address these problems, we have proposed a novel surgical marking system based on radiofrequency identification (RFID) technology, which provides a precise ranging ability. ${ }^{11}$ In our system, $13.56-\mathrm{MHz}$ micro RFID tags are used as "wireless surgical markers" to label lesions that are otherwise difficult to locate. In this study, we test the feasibility and reliability of this RFID marking system using experiments with pseudolesions imitating SPLNs in a canine model.

\section{MATERIAL AND METHODS RFID Marking System}

The schematic diagram of our proposed RFID marking system is shown in Figure 1. The entire system consists of the following 4 main components: (1) micro RFID tags coated with polyester resin (13.56 MHz, $1.0 \times 1.0 \times 0.8 \mathrm{~mm}$; Star Engineering Co, Ltd, Singapore), (2) tag delivery system with bronchoscope, (3) wand-shaped locating probe (10-mm diameter), and (4) signal processing unit with audio interface.

The detailed composition and algorithms of the system have been reported in our previous article. ${ }^{11}$ The micro RFID tag works as a passive transponder without a built-in battery, with the locating probe acting as both a power supply coil and a receiver antenna. When the probe is placed in close proximity to the tag, the tag is activated by the electromagnetic field produced by the probe. The activated tag returns the response signal with a fixed wavelength of $13.56 \pm 0.423 \mathrm{MHz}$. The distance between the probe and the target tag is measured by signal strength and visually presented on a monitor. The signal strength is also converted to a corresponding audio tone by the signal processing units. The operator can then accurately locate the implanted tags by scanning the target area with the probe and reacting to changes in the tone; when the probe is placed closer to the tag, the system produces a higher pitch. The prototype system used in this animal experiment had an effective range of measurement of $7 \mathrm{~mm}$, and the distance can be measured down to the millimeter level.

\section{Animal Care}

Animal care, housing, and surgery were performed with the approval of the Committee for Animal Research of Kyoto University, Japan, which ensures the humane treatment of laboratory animals in compliance with guidelines established by the Ministry of Education, Culture, Sports, Science and Technology, Japan. Seven dogs (age, $<2$ years; body weight, $7.5-12.5 \mathrm{~kg}$ ) were used in this study. Before the bronchoscopic procedures, all dogs were premedicated by intramuscular injection of $0.05 \mathrm{mg} / \mathrm{kg}$ atropine sulfate. They were then sedated with intramuscular injection of $15 \mathrm{mg} / \mathrm{kg}$ ketamine hydrochloride and $3 \mathrm{mg} / \mathrm{kg}$ xylazine hydrochloride. Local anesthesia of the upper respiratory tract was performed using a $1 \%$ lidocaine solution. For surgical procedures, dogs were intubated endotracheally under the same premedication and anesthetic sedation. Sevoflurane and nitrous gas were used for maintenance of anesthesia, under mechanical ventilation. Electrocardiogram and percutaneous oxygen saturation were monitored throughout the surgical procedures.

\section{Preparation of SPLN-Imitating Pseudolesions and Selection of Target Lesions}

Before the operation, pseudolesions imitating SPLNs were created by injecting $0.2 \mathrm{~mL}$ of colored collagen to lung peripheral parenchyma in a selected variety of lobes under CT guidance (Aquilion TSX-101A; Toshiba, Tokyo, Japan). The colored collagen solution was prepared as a mixture of $5 \mathrm{~mL}$ of $1 \%$ collagen solution and $5 \mathrm{~mL}$ of dye $(5 \%$ indocyanine green or indigocarmine). A 23-gauge injection needle (NM-201L-0423; Olympus Corporation, Tokyo, Japan) was used for injecting the solution through the working channel of the bronchoscope (BF240; Olympus). A postprocedure CT scan was performed to confirm the size and location of each created lesion. Peripherally located pseudolesions with a diameter of approximately $10 \mathrm{~mm}$ were selected as target lesions for further experimentation.

\section{Marking of the Target Lesions}

The target lesions were marked within 2 days after creation for the subsequent operative procedures. Under a CT scan guide, an RFID tag was delivered into a small peripheral airway close to each target lesion. Through the 2-mm working channel of the bronchoscope, an introducer tube with a radiopaque marker on its distal tip was navigated to the target lesion (Figure 2). The tube was constructed from polytetrafluoroethylene (Teflon; DuPont Corporation, Wilmington, Del) and has an outer diameter of 1.8 $\mathrm{mm}$. When the operator confirmed that the tip of the introducer tube was in the vicinity of the target lesion, the tag could then be implanted: the RFID tag was inserted from the proximal end of the tube and pushed out from the distal end by a flexible pusher-wire made of stainless steel. A postprocedure CT scan was performed to measure the distance from the tag to the nearest edge of the target lesion.

\section{Operative Procedure}

Immediately after tag delivery to the target lesions, the dogs were brought into an operating room and placed in a lateral decubitus position. With the animals under general anesthesia with intubation and ipsilateral lung collapse, the embedded tag and wedge resection of the target lesion were detected. Three ports with a $10-\mathrm{mm}$ incision were set for each tag -1 for the scope and 2 for port access to the pleural cavity. Under thoracoscopic view, the lobe of interest was scanned with the locating probe to find the target lesion labeled with the tag. When the tag was localized, ringshaped forceps were introduced from the other port to grasp the pleura just 

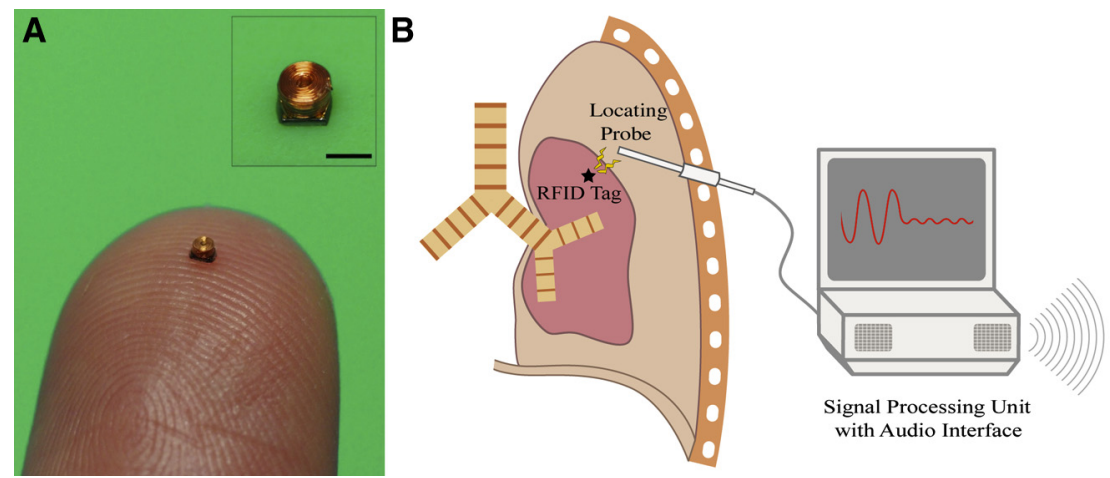

FIGURE 1. A, Photograph of a micro radiofrequency identification (RFID) tag on a forefinger (scale bar $=1 \mathrm{~mm}$ ). The tag works as a passive transponder when it is activated by a radio wave of $13.56 \mathrm{MHz}$. B, Schematic diagram of the RFID marking system, including a wand-shaped locating probe for endoscopic use and a signal processing unit with an audio interface. The operator can locate the implanted tags by scanning the target area with the probe and responding to audio cues; when the probe is nearer the tag, the system produces a higher pitch.

above the site. After a reconfirmation of the tag position, the locating probe was pulled off and an endostapler was introduced from the same port to clamp the estimated resection line. Before the endostapler was fired, the locating probe was reinserted in place of the ring forceps to establish that the tag was contained within the lung tissue to be excised (Figure 3). Owing to the millimeter-level ranging capability of the system, an appropriate surgical margin could be ensured. When the lesion and the tag were resected with the endostaplers, the specimen was incised to measure the surgical margin.

\section{Outcome Measures}

The primary end point of this study was the rate of successful wedge resection of pseudolesions with good margins. Secondary measures were (1)

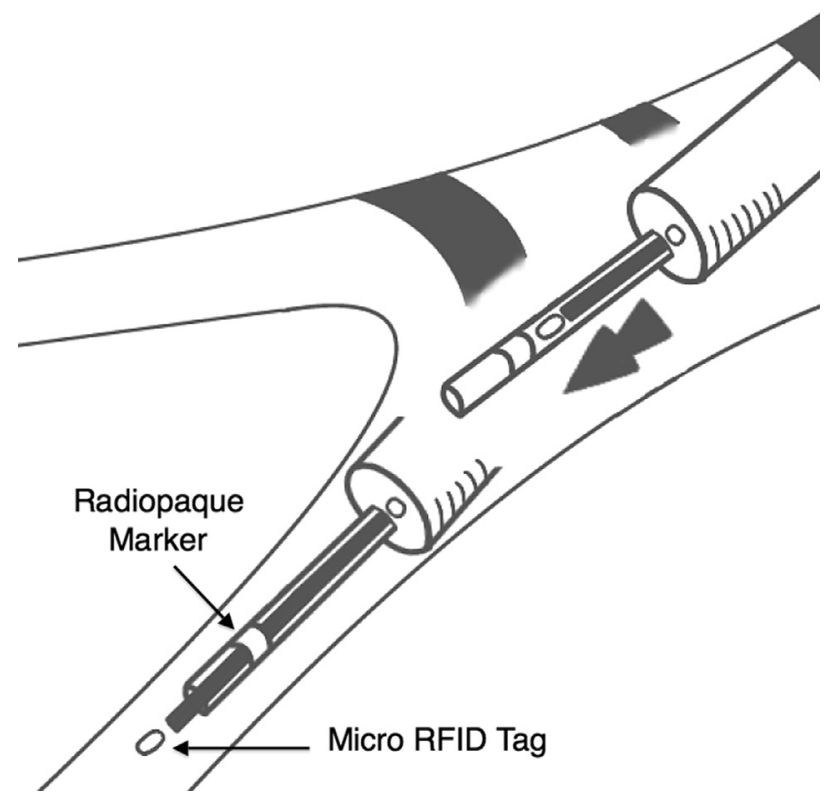

FIGURE 2. Illustration of the radiofrequency identification (RFID) tag delivery system. An introducer tube with a radiopaque marker on its distal tip is inserted through the 2-mm channel of a bronchoscope. The material of the tube is polytetrafluoroethylene (Teflon). An RFID tag is inserted from the proximal end of the tube and pushed out from the distal end by a pusher-wire. the distance from the pseudolesion to the tag as shown in the confirmation $\mathrm{CT}$, (2) the time required for the marking procedure, (3) the time required for tag detection at operation, and (4) the distance from the edge of the pseudolesion to the stapler line, which provides information on the surgical margins (Table 1). The median values and range were calculated for these measures. Finally, we analyzed the system's merits and pitfalls.

\section{RESULTS}

A total of 14 pseudolesions were created by colored collagen injection in 7 dogs. Of the 14 pseudolesions, 10 had a diameter of approximately $10 \mathrm{~mm}$ and were selected as target lesions for further experimentation; there were 2 pseudolesions located in the right cranial lobes, 4 in the right caudal lobes, and 4 in the left caudal lobes. The other 4 pseudolesions were excluded because of larger diffusion or intrabronchial spread of injected collagen solution.

By means of the bronchoscopic marking procedures, each selected pseudolesion was accurately labeled with 1

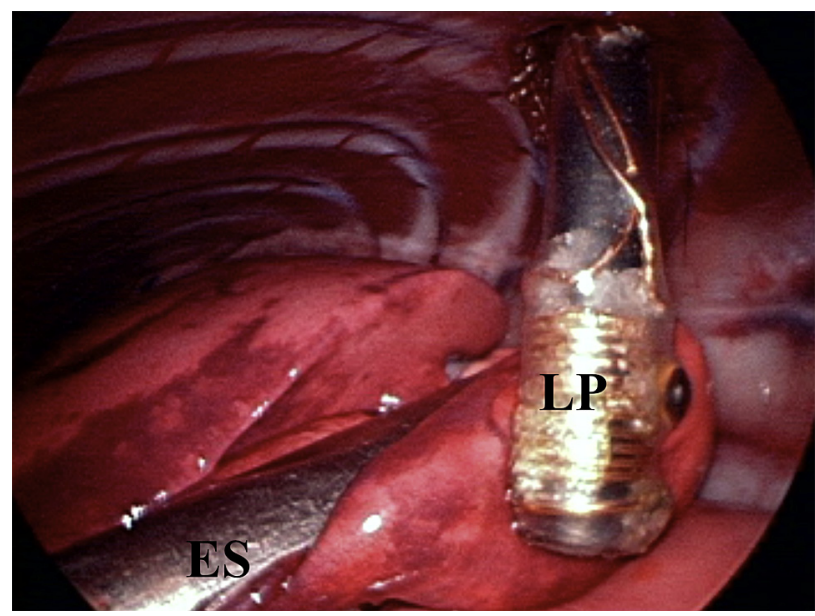

FIGURE 3. Thoracoscopic view of tag detection with a locating probe $(L P)$ and resection of a pseudolesion in peripheral lung parenchyma. Before firing off the endostapler (ES), the operator can confirm the position of the implanted tag to ensure an appropriate margin. 
TABLE 1. Results of the primary end point and secondary outcome measures of the experiments

\begin{tabular}{llc}
\hline \multicolumn{1}{c}{ Primary end point } & & \\
\hline Successful tag detection* & $9 / 10$ & \\
Successful wedge resection* & $9 / 10$ & \\
\hline \multicolumn{1}{c}{ Secondary measures } & & Median \\
\hline Time required for marking (min) & $5-34$ & 11 \\
Distance from the lesion to the tag (mm) & $0-6.5$ & 2.1 \\
Time required for tag detection $(\mathrm{s}) \dagger$ & $10-105$ & 27 \\
Surgical margin $(\mathrm{mm}) \dagger$ & $8-12$ & 11 \\
\hline
\end{tabular}

*One failure was due to dislocation of the tag. $\dagger n=9$.

RFID tag (Figure 4). The tags were placed at a median distance of $2.1 \mathrm{~mm}$ from the lesions (range, $0-6.5 \mathrm{~mm}$, confirmed by postprocedure CT scan). The time required for the marking procedure ranged from 5 to 34 minutes (median, 11 minutes) from the insertion of the bronchoscope to the completion of the confirmation CT scan. Marking procedures were performed just after the creation and selection of the first 3 target pseudolesions and 1 or 2 days after creation for the other 7. No major complications such as pneumothorax or bleeding were observed.

During the thoracoscopic surgical procedures, 9 of the 10 implanted tags were successfully detected by the system. The time required for detection ranged from 10 to $105 \mathrm{sec}-$ onds (median, 27 seconds). Subsequently, wedge resections were performed for these 9 lesions with a median margin of $11 \mathrm{~mm}$ (range, 8-12 mm). In the single nondetected case, a right upper lobectomy was performed only to find no tag in the resected lobe. The dislocated tag was later found in the endotracheal tube.

\section{DISCUSSION}

RFID is a wireless method of automatic identification originally developed for military radar systems in the 1940s to distinguish between friendly and enemy aircraft. ${ }^{12}$
Currently, RFID tags are indispensable in modern society, with applications in item tracking for supply chain management, cashless payments for shopping, and personal identification for ticketing or entrance gates. The continuing miniaturization of RFID tags and development of computer networks have contributed to the widespread use of this system.

Applications have also developed in the field of health care: RFID technology has become widely used for personal identification of medical staff and for tracking drugs and medical equipment in so-called "smart hospitals." 13 Some reports have been published in which RFID technology was deployed to solve problems specific to medical practice. For example, Macario and colleagues ${ }^{14}$ successfully detected surgical sponges labeled with RFID tags in the abdomen, Reicher and colleagues ${ }^{15}$ reported the efficacious monitoring of the position of an RFID tag-labeled endotracheal tube at bedside, and Mayse and colleagues ${ }^{16}$ experimentally implanted RFID tags in canine airways to achieve real-time tracking of tumor positions for stereotactic radiotherapy. In all these medical cases, RFID technology was used to detect the position of an embedded tag.

Our idea was to use micro RFID tags as wireless markers for locating SPLNs in thoracoscopic lung surgery. There is currently only a single previous report showing a similar concept in the field of breast surgery. ${ }^{17}$ That study was essentially a proof of concept in a phantom study using large-sized tags $(2 \mathrm{~mm}$ in diameter and 8 or $12 \mathrm{~mm}$ in length) responding to a radiofrequency of $134.2 \mathrm{kHz}$. To our knowledge, there has been no report on animal experiments or clinical trials of the system, although their tags (VeriChip; Verimed, Inc, Fort Lauderdale, Fla) have been approved by the United States Food and Drug Administration for human use. With the further advances in RFID technology, we have used 1-mm RFID tags in this study and confirmed their feasibility in an animal model for lung wedge resection.

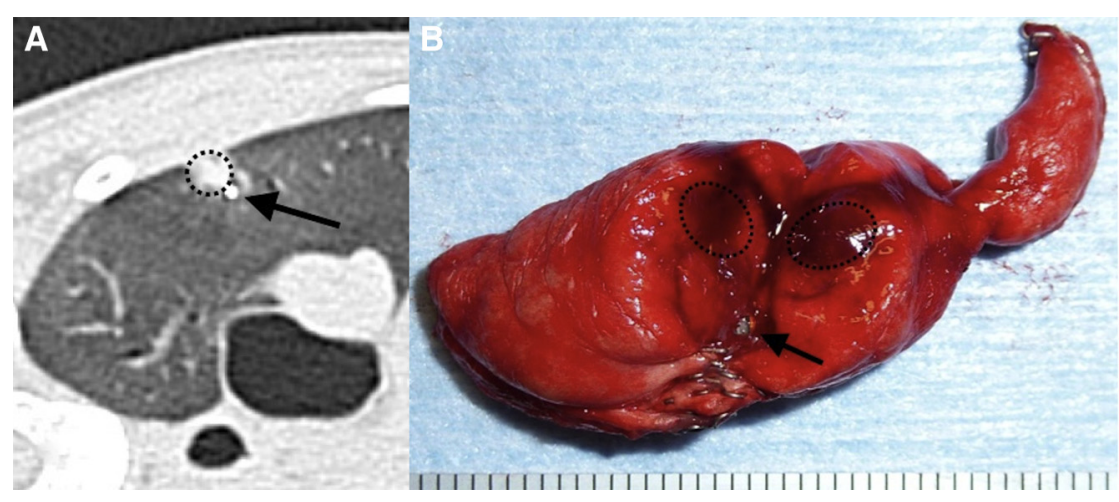

FIGURE 4. A, Computed tomography scan of a canine lung, including a pseudolesion created by colored collagen injection (areas surrounded by black dotted line) and an implanted micro radiofrequency identification tag (black arrow). B, Photograph of a corresponding surgical specimen (scale bar $=1 \mathrm{~mm})$. 
Locating an SPLN has been a challenging task since the era of open thoracotomies. It is even more difficult in a thoracoscopic situation because finger palpation is far less effective. As a consequence, many techniques to assist in the localization of SPLNs have been innovated, with these techniques broadly belonging to 1 of 2 completely different strategies: intraoperative techniques (eg, finger palpation and ultrasound) and preoperative placement of a marker (eg, hook-wire, dye, contrast medium, and radiotracer). ${ }^{4}$

Intraoperative techniques have the virtue of noninvasiveness, and to our knowledge no major complications as a result of these techniques have been reported. Even though intraoperative US is more effective than finger palpation, with a success rate of $70 \%$ to $100 \%$ in selected patients, ${ }^{4}$ this technique has several limitations. ${ }^{5}$ Successful localization using intraoperative US is highly dependent on operator skill. Also, localization is difficult in cases of emphysematous change or incomplete collapse of the target lung. Success rates are further reduced in cases of small nodules less than $1 \mathrm{~cm}$ in size or deep lesions more than $1 \mathrm{~cm}$ from the visceral pleura. Soft nodular ground-glass opacities observed in bronchoalveolar cell carcinomas are also difficult to localize using US. Because of these limitations, intraoperative US has little versatility as a localization technique.

The use of markers is a viable option for surgeons to overcome the limitations of intraoperative localization techniques. The implantation or delivery procedures of several kinds of markers can be classified into 2 categories according to their approaches: a percutaneous pneumocentesis approach and a transbronchial approach with a bronchoscope. Generally, percutaneous implantation of a marker is highly associated with complications such as pneumothorax $(8 \%-$ $50 \%)$, pulmonary hemorrhage $(12 \%-35 \%)$, and pleuritic pain $(5 \%-6 \%){ }^{4}$ In addition, several authors have raised caution on the risk of fatal air embolisms induced by hook-wire implantation. ${ }^{18}$ In contrast, the transbronchial approach can theoretically eliminate the risk of complications related to the percutaneous approach. In our experiments, no major complications were observed related to bronchoscopic delivery of RFID tags, and the time required for marking ranged from 5 to 34 minutes (median, 11 minutes). Bronchoscopic procedures can be a distressing experience for patients, but the transbronchial approach is less invasive than the percutaneous approach.

However, some markers have been used despite their negative aspects. Time constraints are the main cause of failure in the case of dye or radiotracer marking, as the diffusion of dyes and the decay of radiotracers prevent surgeons from successful localization of SPLNs. These shortcomings also lead to difficulties in scheduling preoperative procedures and surgical resection. It has been recommended that surgery be performed within 24 hours after injection of dye and within 12 hours after radiotracer administration. ${ }^{4,5}$ The RFID marking system is free from these time constraints and can provide flexibility in the time interval between the marking procedure and surgery.

In the context of intraoperative fluoroscopy, a contrast medium (ie, barium or lipiodol) is consistently reported as the most effective and reliable marker: Watanabe and colleagues ${ }^{19}$ reported a success rate of $100 \%$ from their experience of 174 nodules; Asano and associates ${ }^{20}$ emphasized a success rate of $100 \%$ for 31 pure ground-glass nodules less than $10 \mathrm{~mm}$ in size; and Okumura and coworkers ${ }^{21}$ stated that good surgical margins could be assured with this technique and that multiple markings can be achieved by transbronchial injection of barium with minimum invasiveness. Unfortunately, despite these merits, the radiation exposure for operators and patients cannot be overlooked. Unnecessary exposure to medical radiation should be avoided if possible, and the use of RFID technology presents an alternative for the purpose of SPLN localization.

The RFID marking system has several positive aspects. The first merit is that of quick detection with simple operability. The system requires only a locating probe in the surgical field. During thoracoscopic surgical procedures, localization of the RFID tag was promptly achieved without the need for unwieldy instruments such as a fluoroscope. As shown in our experiments, the time required for tag detection after insertion of the locating probe ranged from 10 to 105 seconds (median, 27 seconds). Quick detection of the tag and swift localization of the target lesion were achieved by simply listening and reacting to the audio cues from the system; there was no need for operators to shift their gaze from the surgical field or the monitor for the thoracoscope. The simple operability of the system also exempts thoracic surgeons from having to become skilled in an unfamiliar technique such as US interpretation or from the threat of radiation exposure. The second and more important merit is that the $1-\mathrm{mm}$ small wireless marker enables the pinpoint localization of target lesions; the system provides millimeter-level ranging capability to enhance localization accuracy. As a consequence, an appropriate distance from the lesion to the resection line is ensured because the operator can confirm that the RFID marker is included within the lung parenchyma to be resected. In our study with pseudolesions, surgical margins of resected specimens ranged from 8 to $12 \mathrm{~mm}$ (median, $11 \mathrm{~mm}$ ). Accurate localization enables thoracic surgeons to perform sublobar resections with reduced risk of local recurrence in a short procedure time. Third, the labeling of multiple lesions or multiple marking of a single lesion can be achieved in this system because each tag has its own unique identifiable number.

This study has the following limitations. Our prototype system has an effective communication range of $7 \mathrm{~mm}$, mainly owing to limitations in the power supply from the locating probe. Although $7 \mathrm{~mm}$ is adequate for animal experiments, an effective range of $30 \mathrm{~mm}$ may be required 
for clinical use in human lungs. Improvements to the effective range are in progress and can be achieved with further customization of the tag and the probe.

The second limitation is that dislocation of the implanted tag is a problem of critical importance for the RFID marking system. The reason for the sole detection failure in our experiments was the dislocation of the tag to the central airway. To address this problem, we are developing a tag anchoring function. For the purpose of lung tumor tracking during radiation therapy, the use of an RFID tag with anchoring legs was reported to have an $87 \%$ successful stabilization rate for a period of 60 days. ${ }^{22}$ In addition to the anchoring function, the coating of RFID tags is another modification under consideration owing to the possibility of accidental ingestion of a dislocated tag into the digestive tract.

The third limitation is related to the safety of the system. We did not encounter any instances of adverse effects caused by the tag, but the study period was limited to 3 days at its longest. The safety of the in vivo use of RFID tags has been endorsed by long-term experiments in a canine subcutaneous implantation model, ${ }^{23}$ as well as by routine use in livestock farming. Although we have had limited experience with RFID tag placement into a small airway, Mayse and coworkers ${ }^{16}$ have reported no complications in similar experimental implantation of 60 days. To our knowledge, no other published report has described the long-term implantation effects of RFID tags into airways. Further investigation is therefore required into the safety of this system.

The additional cost, time, and radiation exposure could be a demerit of this new method. However, the preoperative marking procedure under CT guidance may be appropriate for cases in which the target is a small peripheral lesion that is difficult to locate under fluoroscopy. RFID tags in commercial mass production are expected to be similar in cost to conventional markers including hook-wires or dye. In general, we estimate that the additional cost, time, and radiation exposure for the RFID marking method are expected to be in the same range as conventional methods that require CT guidance. A cost-benefit analysis is required to determine the best role and use of such techniques in the management of small peripheral lung nodules.

\section{CONCLUSIONS}

The feasibility of our RFID marking system for SPLN localization was demonstrated in a canine model. Further developmental work is underway to improve the effective range, to equip the tags with an anchoring function, and to confirm the safety in implantation.

We are indebted to Mr Tominaga Yukio (Star Engineering Co Ltd, Hitachi, Japan) for his technical help in preparing micro RFID tag. We also thank Miss Mani Sato for preparing nice illustrations.

\section{References}

1. Landreneau RJ, Mack MJ, Dowling RD, Luketich JD, Keenan RJ, Ferson PF, et al. The role of thoracoscopy in lung cancer management. Chest. 1998;113: $6 \mathrm{~S}-12 \mathrm{~S}$

2. Kuwano H, Amano J, Yokomise H. Thoracic and cardiovascular surgery in Japan during 2010: annual report by The Japanese Association for Thoracic Surgery Gen Thorac Cardiovasc Surg. 2012;60:680-708.

3. Boffa DJ, Kosinski AS, Paul S, Mitchell JD, Onaitis M. Lymph node evaluation by open or video-assisted approaches in 11,500 anatomic lung cancer resections. Ann Thorac Surg. 2012;94:347-53; discussion 353.

4. Sortini D, Feo C, Maravegias K, Carcoforo P, Pozza E, Liboni A, et al. Intrathoracoscopic localization techniques. Review of literature. Surg Endosc. 2006;20 1341-7.

5. Daniel TM. A proposed diagnostic approach to the patient with the subcentimeter pulmonary nodule: techniques that facilitate video-assisted thoracic surgery excision. Semin Thorac Cardiovasc Surg. 2005;17:115-22.

6. Aberle DR, Adams AM, Berg CD, Black WC, Clapp JD, Fagerstrom RM, et al. Reduced lung-cancer mortality with low-dose computed tomographic screening. N Engl J Med. 2011;365:395-409.

7. Jaklitsch MT, Jacobson FL, Austin JH, Field JK, Jett JR, Keshavjee S, et al. The American Association for Thoracic Surgery guidelines for lung cancer screening using low-dose computed tomography scans for lung cancer survivors and other high-risk groups. J Thorac Cardiovasc Surg. 2012;144:33-8.

8. Suzuki K, Nagai K, Yoshida J, Ohmatsu H, Takahashi K, Nishimura M, et al. Video-assisted thoracoscopic surgery for small indeterminate pulmonary nodules: indications for preoperative marking. Chest. 1999;115:563-8.

9. Schuchert MJ, Abbas G, Awais O, Pennathur A, Nason KS, Wilson DO, et al. Anatomic segmentectomy for the solitary pulmonary nodule and early-stage lung cancer. Ann Thorac Surg. 2012;93:1780-5.

10. Welter S, Theegarten D, Trarbach T, Maletzki F, Stamatis G, Tötsch M. Safety distance in the resection of colorectal lung metastases: a prospective evaluation of satellite tumor cells with immunohistochemistry. J Thorac Cardiovasc Surg. 2011;141:1218-22

11. Takahata H, Kojima F, Okada M, Sugiura T, Sato T, Oshiro O. Thoracoscopic surgery support system using passive RFID marker. In: Engineering in Medicine and Biology Society (EMBC). 2012 Annual International Conference of the IEEE 2012:183-186.

12. Stockman H. Communication by means of reflected power. Proc IRE. 1948;36: 1196-204.

13. Fuhrer P, Guinard D. Building a Smart HospitNonEning RFID technologies. European Conference on eHealth. 2006;5.

14. Macario A, Morris D, Morris S. Initial clinical evaluation of a handheld device for detecting retained surgical gauze sponges using radiofrequency identification technology. Arch Surg. 2006;141:659-62.

15. Reicher J, Reicher D, Reicher M. Use of radio frequency identification (RFID) tags in bedside monitoring of endotracheal tube position. J Clin Monit Comput. 2007;21:155-8.

16. Mayse ML, Parikh PJ, Lechleiter KM, Dimmer S, Park M, Chaudhari A, et al Bronchoscopic implantation of a novel wireless electromagnetic transponder in the canine lung: a feasibility study. Int J Radiat Oncol Biol Phys. 2008;72:93-8.

17. Reicher JJ, Reicher MA, Thomas M, Petcavich R. Radiofrequency identification tags for preoperative tumor localization: proof of concept. AJR Am J Roentgenol. 2008;191:1359-65

18. Sakiyama S, Kondo K, Matsuoka H, Yoshida M, Miyoshi T, Yoshida S, et al. Fatal air embolism during computed tomography-guided pulmonary marking with a hook-type marker. $J$ Thorac Cardiovasc Surg. 2003;126:1207-9.

19. Watanabe K, Nomori H, Ohtsuka T, Kaji M, Naruke T, Suemasu K. Usefulness and complications of computed tomography-guided lipiodol marking for fluoroscopy-assisted thoracoscopic resection of small pulmonary nodules: experience with 174 nodules. J Thorac Cardiovasc Surg. 2006;132:320-4.

20. Asano F, Shindoh J, Shigemitsu K, Miya K, Abe T, Horiba M, et al. Ultrathin bronchoscopic barium marking with virtual bronchoscopic navigation for fluoroscopy-assisted thoracoscopic surgery. Chest. 2004;126:1687-93.

21. Okumura T, Kondo H, Suzuki K, Asamura H, Kobayashi T, Kaneko M, et al. Fluoroscopy-assisted thoracoscopic surgery after computed tomography-guided bronchoscopic barium marking. Ann Thorac Surg. 2001;71:439-42.

22. Shah AP, Kupelian PA, Willoughby TR, Meeks SL. Expanding the use of realtime electromagnetic tracking in radiation oncology. J Appl Clin Med Phys. 2011;12:3590.

23. Murasugi E, Koie H, Okano M, Watanabe T, Asano R. Histological reactions to microchip implants in dogs. Vet Rec. 2003;153:328-30. 